\title{
Article \\ Cerebral Autoregulation during Postural Change in Patients with Cervical Spinal Cord Injury-A Carotid Duplex Ultrasonography Study
}

\author{
Joo-Hyun Kee ${ }^{1} \mathbb{D}$, Jun-Hyeong Han ${ }^{1}$, Chang-Won Moon ${ }^{1}$ and Kang Hee Cho ${ }^{1,2, *(\mathbb{D})}$ \\ 1 Department of Rehabilitation Medicine, School of Medicine, Chungnam National University, \\ Daejeon 35015, Korea; awngusla@naver.com (J.-H.K.); jhhan1125@cnuh.co.kr (J.-H.H.); \\ mcw0171@cnuh.co.kr (C.-W.M.) \\ 2 Department of Biomedical Institute, Chungnam National University, Daejeon 35015, Korea \\ * Correspondence: khcho@cnu.ac.kr
}

Citation: Kee, J.-H.; Han, J.-H.; Moon, C.-W.; Cho, K.H. Cerebral Autoregulation during Postural Change in Patients with Cervical Spinal Cord Injury-A Carotid Duplex Ultrasonography Study. Diagnostics 2021, 11, 1321. https:// doi.org/10.3390/diagnostics11081321

Academic Editor: Michael Henein

Received: 19 June 2021

Accepted: 21 July 2021

Published: 23 July 2021

Publisher's Note: MDPI stays neutral with regard to jurisdictional claims in published maps and institutional affiliations.

Copyright: (c) 2021 by the authors. Licensee MDPI, Basel, Switzerland. This article is an open access article distributed under the terms and conditions of the Creative Commons Attribution (CC BY) license (https:// creativecommons.org/licenses/by/ $4.0 /)$.

\begin{abstract}
Patients with a spinal cord injury (SCI) frequently experience sudden falls in blood pressure during postural change. Few studies have investigated whether the measurement of blood flow velocity within vessels can reflect brain perfusion during postural change. By performing carotid duplex ultrasonography (CDU), we investigated changes in cerebral blood flow (CBF) during postural changes in patients with a cervical SCI, determined the correlation of CBF change with presyncopal symptoms, and investigated factors affecting cerebral autoregulation. We reviewed the medical records of 100 patients with a cervical SCI who underwent CDU. The differences between the systolic blood pressure, diastolic blood pressure, and CBF volume in the supine posture and after $5 \mathrm{~min}$ at $50^{\circ}$ tilt were evaluated. Presyncopal symptoms occurred when the blood flow volume of the internal carotid artery decreased by $\geq 21 \%$ after tilt. In the group that had orthostatic hypotension and severe CBF decrease during tilt, the body mass index and physical and functional scores were lower than in other groups, and the proportion of patients with a severe SCI was high. The higher the SCI severity and the lower the functional score, the higher the possibility of cerebral autoregulation failure. CBF should be assessed by conducting CDU in patients with a high-level SCI.
\end{abstract}

Keywords: cervical spinal cord injury; cerebral autoregulation; orthostatic hypotension; cerebral blood flow; carotid duplex ultrasonography

\section{Introduction}

Significant cardiovascular and autonomic dysfunction is a common consequence of high-level spinal cord injuries (SCIs) [1]. Patients with an SCI frequently experience a sudden reduction in blood pressure (BP) upon postural change, which is characterized by dizziness, light-headedness, or even syncope. Orthostatic hypotension $(\mathrm{OH})$ is very difficult to control and severely impairs the quality of life [2]. The symptoms associated with $\mathrm{OH}$ significantly interfere with the rehabilitation of patients with an SCI. BP reduction that is diagnostic of $\mathrm{OH}$ occurs in $74 \%$ of SCI patients, and symptoms of $\mathrm{OH}$ (such as lightheadedness or dizziness) occur in 59\% of individuals with an SCI during physiotherapy [3].

Possible mechanisms underlying $\mathrm{OH}$ in SCI patients include changes in sympathetic activity, altered baroreflex function, lack of skeletal muscle pumping activity, cardiovascular deconditioning, and altered salt and water balance [4,5]. However, some people are relatively insensitive to low BP and can maintain consciousness despite low arterial pressure [4]. In these individuals, despite low perfusion pressure, there is a change in cerebral autoregulation (CA) that maintains cerebral blood flow (CBF). Therefore, $\mathrm{CA}$, and not systemic $\mathrm{BP}$, is the predominant factor responsible for the symptoms of $\mathrm{OH}$ [6].

Few studies have investigated whether the measurement of blood flow velocity within vessels can reflect brain perfusion during postural change. The blood flow velocity in the 
middle cerebral artery (MCA) is highly correlated with CBF under conditions of varying mean arterial pressure and cerebrovascular conductance $[7,8]$. We believe that transcranial Doppler (TCD), which has been mainly used so far, can evaluate cerebral blood flow velocity, but cannot evaluate the cross-sectional area of blood vessels; thus, there is a limit to accurately measuring CBF with TCD. Several studies have shown that the blood flow volume (BFV) of the internal carotid artery (ICA) can be reliably measured, and that it has a close correlation with CBF values [9-11]. A previous study used carotid duplex ultrasonography (CDU) as a tool for CBF measurement in patients with brain injury, and it revealed a significant correlation with BFV compared to when TCD was used [12].

Few studies have measured CBF in patients with an SCI and evaluated cerebral hemodynamics during postural changes. In this study, we assessed CBF by using CDU to measure changes in the internal carotid blood flow during postural changes.

The purpose of this study was to investigate, with CDU, the changes in cerebral blood flow volume (CBFV) after postural change, using a tilt table in patients with tetraplegia due to a cervical spinal cord injury (CSCI). We aimed to confirm the correlation of CBFV with symptoms of $\mathrm{OH}$ and evaluate the necessity of applying this test in actual clinical practice. In addition, we aimed to determine whether the degree of the SCI was related to changes in $\mathrm{CBF}$ when standing, and to elucidate the factors that affect $\mathrm{CA}$.

\section{Materials and Methods}

\subsection{Study Design}

This study was a retrospective review of the medical records of all patients who underwent CDU after a CSCI at the Department of Rehabilitation Medicine, Chungnam National University Hospital, from 1 January 2018 to 31 December 2019.

Patients with complete or incomplete tetraplegia due to a CSCI and who were 18 years of age or older were included in the study. We excluded cases with insufficient medical records or insufficient test results to explain hemodynamic changes, cases with history of fatal cardiovascular disease, and cases of unstable SCIs. Finally, the medical records of 100 patients were reviewed.

\subsection{Protocol of Carotid Duplex Ultrasonography}

We conducted CDU during head-up tilt in patients with a CSCI. The ICA was studied on both sides, and intravascular flow volumes were calculated using a 9-MHz linear array transducer. BFV measurements were automatically calculated by the built-in software of the ultrasound device (Siemens ACUSON, Siemens Healthcare, Erlangen, Germany). For flow-volume measurements, the head was turned $25^{\circ}-40^{\circ}$ to the contralateral side, and a straight segment of the ICA, at least $2 \mathrm{~cm}$ above the carotid bulb, was selected (Figure 1). Measurements were performed on a horizontal segment in the sagittal plane. The arterial diameter was calculated as a vertical line through the lumen between the echogenic intimal layers. The value obtained from the test is volume flow rate, which was automatically calculated from the cross-sectional area of the blood vessel and the time-averaged mean velocity: volume flow rate $=$ area $\left(\mathrm{cm}^{2}\right) \times$ time-averaged mean velocity $(\mathrm{cm} / \mathrm{s})$. The peak systolic velocity, end diastolic velocity, time-averaged mean velocity, vessel diameter, and vessel area were all measured during the test. All patients were allowed to rest on the examination table for $5 \mathrm{~min}$ before the test. CBFV, BP, and heart rate (HR) were measured in the supine position, immediately after the patient was tilted by $50^{\circ}$, and $5 \mathrm{~min}$ after the tilt. To increase the reliability of the test, CBF was measured three times in each position, and the mean value was used. In addition, the presence or absence of presyncopal symptoms, such as dizziness, light-headedness, nausea, and blurry vision, in each position, was recorded. 


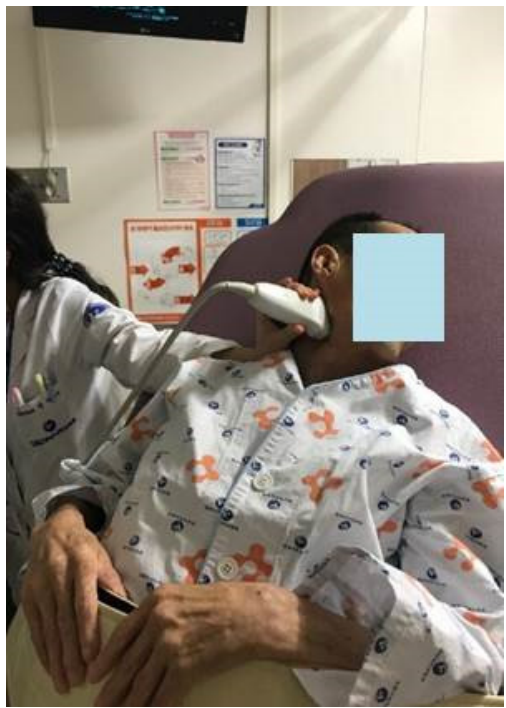

(a)

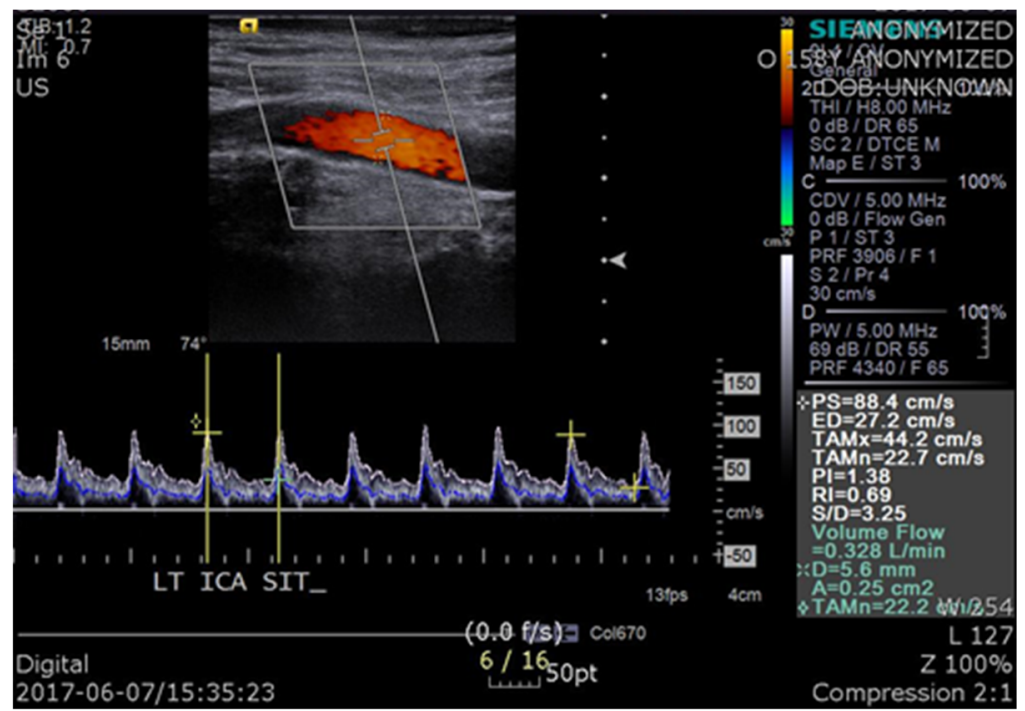

(b)

Figure 1. Carotid duplex sonography: (a) The position of the patient and measurements of cerebral blood flow using carotid duplex ultrasonography; (b) The value of interest in the test was volume flow.

\subsection{Data Acquisition and Data Analysis}

The data obtained during the ICA Duplex ultrasonography were systolic blood pressure (SBP), diastolic blood pressure (DBP), HR, average BFV, and presence of $\mathrm{OH}$ symptoms in each position. In order to determine whether the patient experienced $\mathrm{OH}$ symptoms when BFV (L/min) was reduced to some extent during the tilt, after obtaining the BFV difference $\left(\mathrm{BFV}\right.$ difference $(\%)=\mathrm{BFV}($ supine $)-\mathrm{BFV}\left(\right.$ tilt $50^{\circ}$ or $\left.5 \mathrm{~min}\right) / \mathrm{BFV}($ supine $\left.) \times 100\right)$ between the value measured in the supine posture and the value measured after $5 \mathrm{~min}$ at $50^{\circ}$ of tilt, the point with the highest sensitivity and specificity was obtained by applying the receiver operating characteristic (ROC) curve to the relationship between the BFV difference and the presence of symptoms. It was assumed that there would be symptoms of $\mathrm{OH}$ when $\mathrm{CBF}$ fell below that point.

We calculated the difference between the SBP, DBP, and CBFV values measured in the supine position and the values measured after $5 \mathrm{~min}$ at $50^{\circ}$ tilt. Based on the presence of $\mathrm{OH}$ or $\triangle \mathrm{CBF}$, the patients were divided into four groups. The presence of $\mathrm{OH}$ was determined according to criteria defined by The American Autonomic Society and the American Academy of Neurology (OH was defined as a decrease in SBF of at least $20 \mathrm{~mm}$ $\mathrm{Hg}$ or a decrease in DBF of at least $10 \mathrm{~mm} \mathrm{Hg}$ within three minutes of standing up). If there was a difference in BFV beyond the set point of the presyncopal symptoms mentioned above, it was marked as $\triangle \mathrm{CBF}+$; otherwise, it was marked as $\triangle \mathrm{CBF}-$. As shown in Figure 2, patients in group $1(\mathrm{G} 1)$ had $\mathrm{OH}$ and decreased $\mathrm{CBF}(\mathrm{OH}+, \Delta \mathrm{CBF}+)$, those in group 2 (G2) had $\mathrm{OH}$ but preserved $\mathrm{CBF}(\mathrm{OH}+, \triangle \mathrm{CBF}-)$, those in group $3(\mathrm{G} 3)$ did not have $\mathrm{OH}$ but had decreased $\mathrm{CBF}(\mathrm{OH}-, \triangle \mathrm{CBF}+)$, and those in group $4(\mathrm{G} 4)$ had neither $\mathrm{OH}$ nor decreased $\mathrm{CBF}(\mathrm{OH}-, \triangle \mathrm{CBF}-)$.

Data on age, height, body mass index (BMI), duration of injury (DOI), American Spinal Cord Injury Association impairment scale (AIS) grade, sex, neurological level of injury (NLI), and underlying disease (such as diabetes mellitus (DM) and hypertension (HTN)) were collected in each group. To confirm the relationship between functional status and CA, the motor score (MS), sensory score (SS), and Korean spinal cord independence measure (K-SCIM) score were assessed. In addition, the presence or absence of presyncopal symptoms after $5 \mathrm{~min}$ at $50^{\circ}$ tilt was noted in each group. 


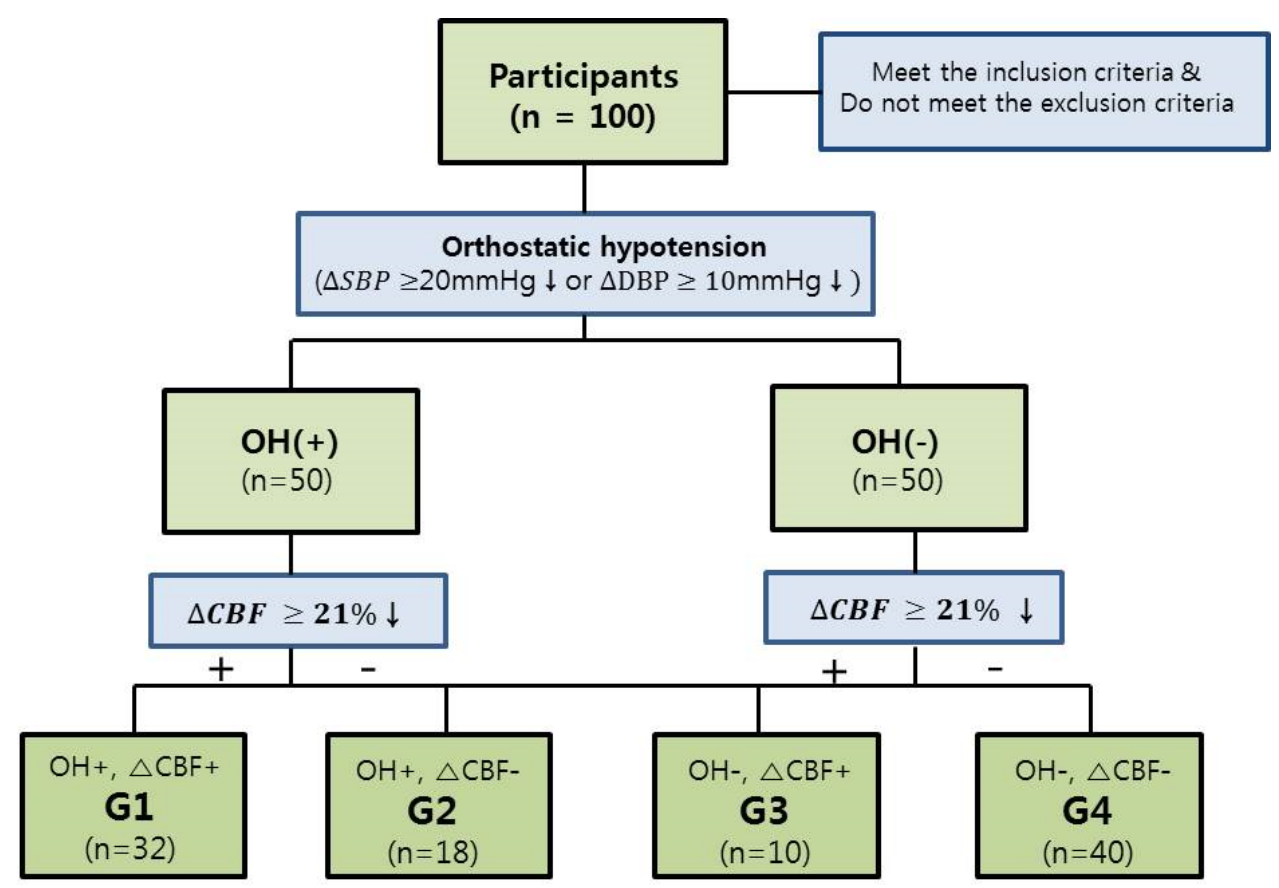

Figure 2. Recruitment of patients and division into groups for data analysis. $\mathrm{OH}$, orthostatic hypotension; CBF, cerebral blood flow; SBP, systolic blood pressure; DBP, diastolic blood pressure.

\subsection{Statistical Analysis}

The ROC curve was used to determine whether the patient experienced symptoms when the BFV $(\mathrm{L} / \mathrm{min})$ decreased to a certain extent at $5 \mathrm{~min}$ after $50^{\circ}$ tilt. A one-way analysis of variance was used to compare differences in age, height, BMI, DOI, MS (upper extremity, UE), MS (lower extremity, LE), total MS, SS (light touch, LT), SS (pin prick, PP), total SS, and K-SCIM among the four groups. For post-analysis, the Scheffe test was used to determine whether the differences among the groups were significant. The data are reported as mean \pm standard error. Crossover analysis and chi-squared test were used to evaluate differences in distributions of AIS grades, sex, NLI, DM, HTN, and the presence of presyncopal symptoms among the four groups. All statistical analyses were performed with IBM Statistical Product and Service Solutions software, version 26.0 (IBM Corporation, Armonk, NY, USA). Statistical significance was set at $p<0.05$.

\section{Results}

3.1. The Relationship between the Decrease in CBFV (L/min) after Tilt and Presence of Presyncopal Symptoms

Of the 100 patients included in the study, 40 complained of presyncopal symptoms during tilt. The ROC curve of the difference in CBFV after tilt in the presence of presyncopal symptoms is shown in Figure 3, and the data are further presented in Table 1. According to the analysis of the ROC curve, presyncopal symptoms occurred when CBFV decrease was more than $21 \%$ after tilt, with a sensitivity of 0.875 and specificity of 0.967 (Table 1). 
ROC curve

for Complaints of presyncopal symptoms

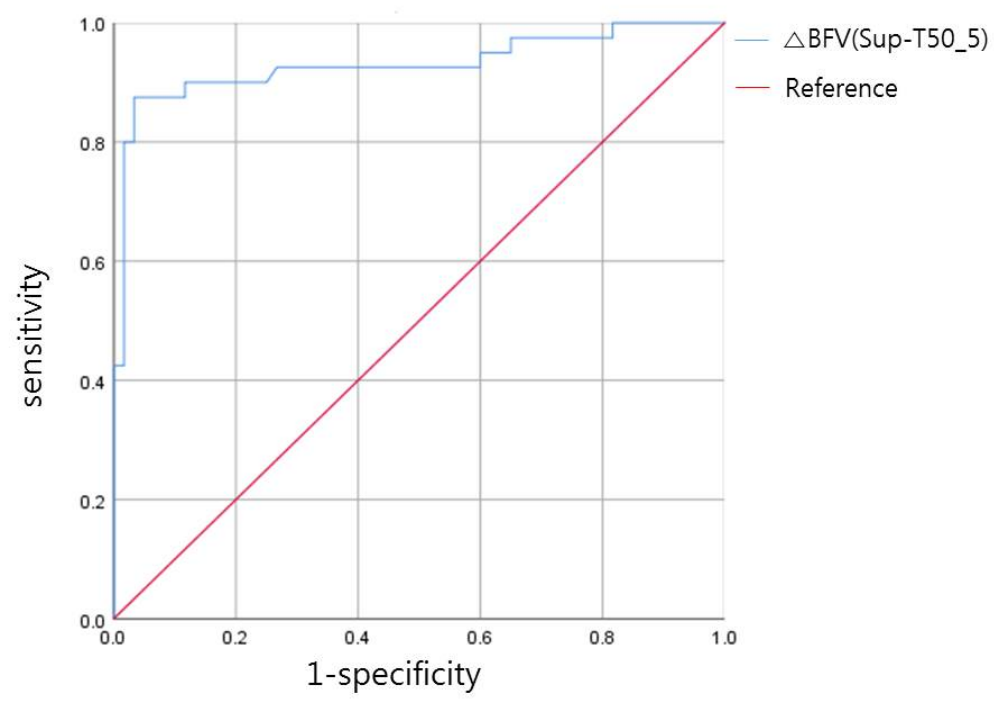

Figure 3. Receiver operating characteristic (ROC) curve for the relationship between decrease in blood flow volume (BFV, L/min) after tilt and the presence of presyncopal symptoms.

Table 1. Decrease in BFV after tilt, resulting in presyncopal symptoms.

\begin{tabular}{|c|c|c|c|c|c|}
\hline & AUC & $95 \%$ CI & Cut-Off Value (\%) & Sensitivity & Specificity \\
\hline$\Delta \mathrm{BFV}$ (Sup-T50_5) & 0.93 & $(0.870 \sim 0.991)$ & 21.23 & 0.875 & 0.967 \\
\hline
\end{tabular}

\subsection{Demographic Data of Groups and Comparison of Characteristics of Each Group}

$\mathrm{G} 1(\mathrm{OH}+, \Delta \mathrm{CBF}+)$ had 32 patients, $\mathrm{G} 2(\mathrm{OH}+, \Delta \mathrm{CBF}-)$ had 18, $\mathrm{G} 3(\mathrm{OH}-, \Delta \mathrm{CBF}+)$ had 10 , and $\mathrm{G} 4(\mathrm{OH}-, \triangle \mathrm{CBF}-)$ had 40 patients, as shown in Figure 2 . There was no statistically significant difference in age, height, and DOI among the groups, but there was a significant difference in BMI among the groups (Table 2). The difference in AIS grades among the groups was also significant (Table 3). In G1, the number of patients with AIS grades A, B, and $C$ was 26 out of $32(81.25 \%)$, which was much higher than that of the other groups. The proportions of the AIS grades in G1 were $92.31 \%$ for AIS grade A (12 out of 13 patients), $75.00 \%$ for AIS grade B ( 3 out of 4 ), $57.89 \%$ for AIS grade C (11 out of 19 ), and $9.38 \%$ for AIS grade D (6 out of 64 ). In G4, $54.69 \%$ of patients ( 35 out of 64 ) had AIS grade D injury, which was higher than the proportion of AIS grades A, B, and C injuries. Sex, NLI, and presence of underlying disease (DM and HTN) were not significantly different among the groups (Table 3 ). When comparing the functional scores among the four groups, there were significant differences in the MS, SS, and K-SCIM scores ( $p$-value $<0.05$, Table 4 ). In multiple comparisons, the average BMI in G4 was $3.095 \mathrm{~kg} / \mathrm{m}^{2}(p=0.006)$ higher than that in G1. MS (UE), MS (LE), MS (total), and K-SCIM scores were lower in G1 than in the other groups, and the differences were statistically significant. SS (LT), SS (PP), and SS (total) values in G1 were also significantly lower than those in G4 $(p<0.05)$. 
Table 2. The demographic data and characteristics of patients in the four groups.

\begin{tabular}{cccccc}
\hline Characteristic, Mean \pm SD & G1 $(\boldsymbol{n}=\mathbf{3 2})$ & G2 $(\boldsymbol{n}=\mathbf{1 8})$ & G3 $(\boldsymbol{n = 1 0 )}$ & G4 $(\boldsymbol{n}=\mathbf{4 0})$ & Total $(\boldsymbol{n}=\mathbf{1 0 0})$ \\
\hline Age $($ years $)$ & $58.5 \pm 16.97$ & $60.61 \pm 14.74$ & $63.00 \pm 18.94$ & $57.22 \pm 13.50$ & $58.82 \pm 15.34$ \\
Height $(\mathrm{cm})$ & $169.41 \pm 7.98$ & $163.63 \pm 9.31$ & $164.18 \pm 13.50$ & $167.20 \pm 8.41$ & $166.96 \pm 9.16$ \\
BMI $\left(\mathrm{kg} / \mathrm{m}^{2}\right)$ & $20.92 \pm 3.44$ & $23.38 \pm 4.54$ & $23.078 \pm 2.60$ & $24.01 \pm 3.49$ & $22.82 \pm 3.80$ \\
DOI & $185.17 \pm 432.04$ & $145.95 \pm 188.82$ & $118.89 \pm 142.34$ & $158.29 \pm 222.59$ & $160.74 \pm 293.85$ \\
\hline
\end{tabular}

BMI, body mass index; DOI, duration of injury; G1, group 1; G2, group 2; G3; group 3; G4, group 4; SD, standard deviation; * $p<0.05$.

Table 3. The demographic data and characteristics of patients in the four groups.

\begin{tabular}{|c|c|c|c|c|c|c|}
\hline & & G1 $(n=32)$ & G2 $(n=18)$ & G3 $(n=10)$ & G4 $(n=40)$ & Total $(n=100)$ \\
\hline \multicolumn{7}{|l|}{ Characteristic } \\
\hline \multirow{4}{*}{ AIS grade $(n) *$} & AIS A & 12 & 0 & 0 & 1 & 13 \\
\hline & AIS B & 3 & 0 & 1 & 0 & 4 \\
\hline & AIS C & 11 & 3 & 1 & 4 & 19 \\
\hline & AIS D & 6 & 15 & 8 & 35 & 64 \\
\hline \multirow[t]{2}{*}{$\operatorname{Sex}(n)$} & Male & 26 & 13 & 8 & 30 & 77 \\
\hline & Female & 6 & 5 & 2 & 10 & 23 \\
\hline \multirow{6}{*}{ NLI $(n)$} & C2 & 1 & 0 & 0 & 1 & 2 \\
\hline & C3 & 4 & 3 & 1 & 2 & 10 \\
\hline & $\mathrm{C} 4$ & 12 & 7 & 5 & 16 & 40 \\
\hline & C5 & 14 & 8 & 4 & 18 & 44 \\
\hline & C6 & 0 & 0 & 0 & 2 & 2 \\
\hline & $\mathrm{C} 8$ & 1 & 0 & 0 & 1 & 2 \\
\hline \multirow[t]{2}{*}{$\operatorname{DM}(n)$} & $(-)$ & 31 & 12 & 7 & 38 & 88 \\
\hline & $(+)$ & 1 & 6 & 3 & 2 & 12 \\
\hline \multirow[t]{2}{*}{$\operatorname{HTN}(n)$} & $(-)$ & 23 & 13 & 7 & 31 & 74 \\
\hline & $(+)$ & 9 & 5 & 3 & 9 & 26 \\
\hline
\end{tabular}

AIS, American Spinal Injury Association Impairment Scale; G1, group 1; G2, group 2; G3; group 3; G4, group 4 ; NLI, neurologic level of injury; DM, diabetes mellitus; HTN, hypertension, ${ }^{*} p<0.05$.

Table 4. Comparison of functional scores among the four groups.

\begin{tabular}{ccccccc}
\hline $\begin{array}{c}\text { Functional Score } \\
(\text { Mean } \pm \text { SD) }\end{array}$ & G1 $(\boldsymbol{n}=\mathbf{3 2})$ & G2 $(\boldsymbol{n}=\mathbf{1 8})$ & G3 $(\boldsymbol{n}=\mathbf{1 0})$ & G4 $(\boldsymbol{n}=\mathbf{4 0 )}$ & Total & $\begin{array}{c}\boldsymbol{p} \text {-Value } \\
(\text { ANOVA) }\end{array}$ \\
\hline MS (UE) & $22.25 \pm 11.92$ & $34.11 \pm 5.95$ & $34.2 \pm 7.56$ & $36.33 \pm 6.60$ & $31.21 \pm 10.59$ & 0.000 \\
MS (LE) & $12.03 \pm 15.23$ & $33.06 \pm 12.71$ & $33.70 \pm 18.28$ & $36.93 \pm 10.07$ & $27.94 \pm 17.12$ & 0.000 \\
MS (total) & $34.28 \pm 23.11$ & $67.17 \pm 15.89$ & $67.90 \pm 22.66$ & $73.25 \pm 14.92$ & $59.15 \pm 25.39$ & 0.000 \\
SS (LT) & $56.28 \pm 22.49$ & $65.56 \pm 13.98$ & $65.30 \pm 19.03$ & $70.78 \pm 20.92$ & $64.65 \pm 20.87$ & 0.032 \\
SS (PP) & $52.66 \pm 25.03$ & $64.00 \pm 13.23$ & $60.80 \pm 9.39$ & $73.23 \pm 23.11$ & $63.74 \pm 22.83$ & 0.002 \\
SS (total) & $108.94 \pm 46.01$ & $129.56 \pm 26.41$ & $126.10 \pm 25.80$ & $144.00 \pm 43.00$ & $128.39 \pm 42.35$ & 0.005 \\
K-SCIM & $23.44 \pm 21.76$ & $62.44 \pm 25.80$ & $57.70 \pm 25.74$ & $64.78 \pm 25.82$ & $50.42 \pm 30.59$ & 0.00000 \\
\hline
\end{tabular}

MS, motor score; UE, upper extremity; LE, lower extremity; SS, sensory score; LT, light touch; PP, pin prick; K-SCIM, Korean spinal cord independence measure; G1, group 1; G2, group 2; G3; group 3; G4, group 4; ANOVA, analysis of variance.

\subsection{The Incidence of Presyncopal Symptoms in Each Group}

Presyncopal symptoms were found in approximately $96.88 \%$ of patients in G1, 5.56\% in G2, 40\% in G3, and 10\% in G4 (Figure 4). In G2, even if there was a drop in BP during tilt, since $\mathrm{CBF}$ was maintained, symptoms rarely occurred. 


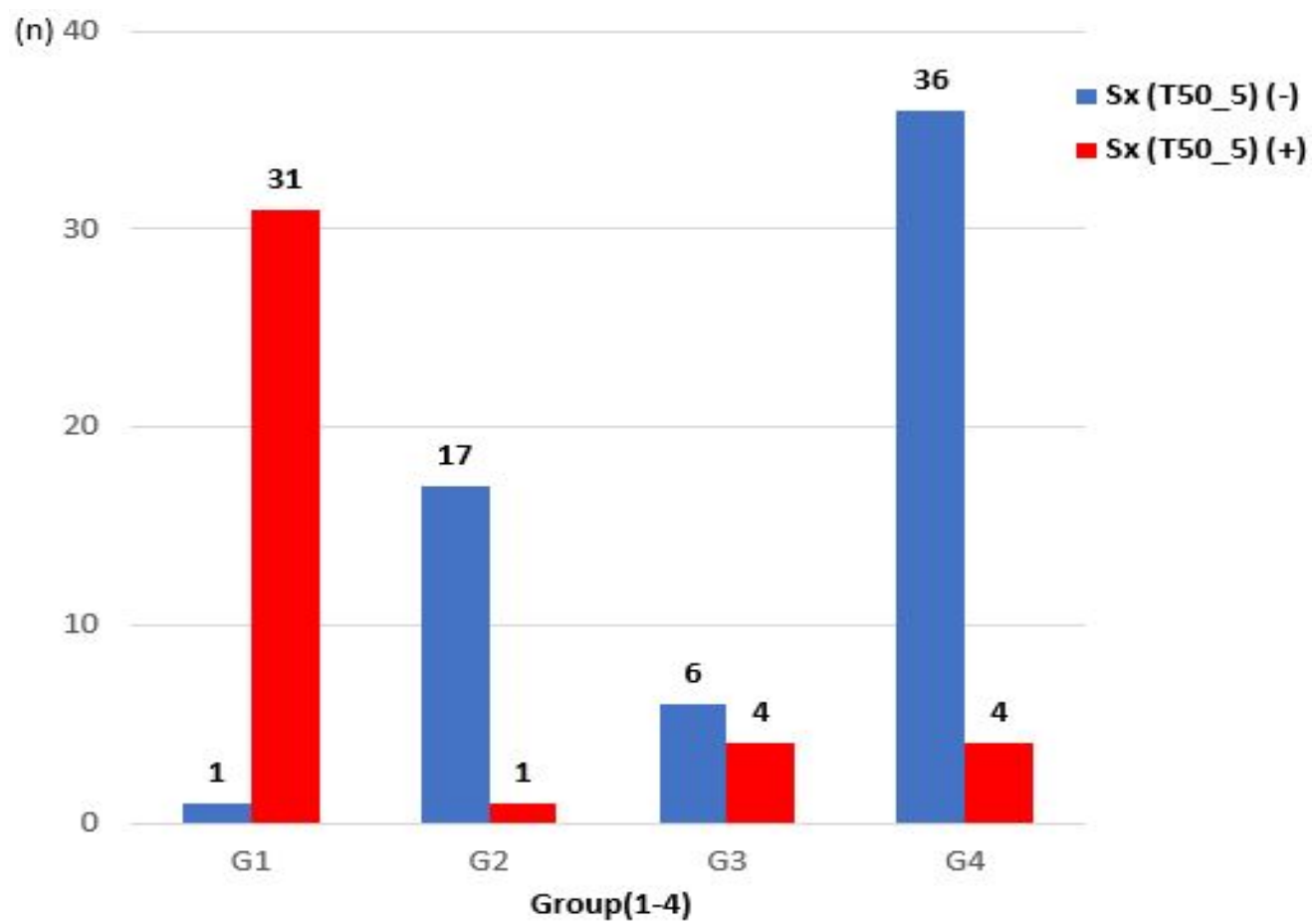

Figure 4. Comparison of the occurrence of presyncopal symptoms among groups.

\section{Discussion}

To our knowledge, this is the first study to use CDU to investigate changes in CBF during postural change in patients with a CSCI. The main findings are as follows:

1. Presyncopal symptoms occurred when the BFV of the ICA decreased by $\geq 21 \%$ after tilt in patients with a CSCI.

2. In G1, i.e., patients who had $\mathrm{OH}$ and severe $\mathrm{CBF}$ decrease during tilt (because $\mathrm{CA}$ did not occur), the BMI was lower than that in G4 (patients who had neither OH nor CBF decrease); physical and functional scores such as MS (UE), MS (LE), MS (total), SS (LT), SS (PP), SS (total), and K-SCIM scores were low; and the proportions of AIS grades $\mathrm{A}, \mathrm{B}$, and $\mathrm{C}$ were high.

3. In G2, i.e., the group of patients who had no decrease in $\mathrm{CBF}$ even though there was $\mathrm{OH}$ during tilt (because $\mathrm{CA}$ occurred), presyncopal symptoms rarely occurred (5.56\%).

The mechanism that underlies $\mathrm{OH}$ after an SCI remains unclear. According to some studies, if there is a problem in the pathway from the motor center to the sympathetic nerves due to a high-level SCI, the activation of the sympathetic nervous system through baroreceptors and chemoreceptors fails. Therefore, when external factors that can affect BP, such as postural changes, occur, the mechanism of maintaining normal BP does not work. Therefore, in the early stages of an $\mathrm{SCI}, \mathrm{OH}$ is a result of decreased sympathetic nerve response, venous vasodilation, abdominal muscle paralysis, and inadequate secretion of hormones $[4,13]$. However, over time, the distal sympathetic preganglionic neuronal conduction pathways of patients with an $\mathrm{SCI}$ adapt, and $\mathrm{OH}$ improves through the partial recovery of sympathetic nerve function, increased secretion of vasoconstrictors, and increased susceptibility of blood vessels to these vasoconstrictors [14]. In addition, studies have shown that compensation through the kidneys is an important part of the long-term adaptation mechanism for sympathetic nervous system abnormalities [15]. Another study reported increased sensitivity to arginine vasopressin in patients with an SCI [16]. On the other hand, animal experiments in one study showed that neither the sympathetic nervous system nor arginine vasopressin had any effect on BP fluctuations after an SCI [17].

In 1991, a study using TCD suggested that the difference in CA, which regulates CBF, rather than changes in BP, would be involved in symptomatic improvement in patients with sympathetic nervous system abnormalities due to an SCI [6]. Since then, several 
studies have investigated CBF control in patients with a high-level SCI using TCD [18,19]. CA involves myogenic, metabolic, and neurogenic control mechanisms, as well as systemic factors [20,21], and while static CA is well preserved in patients with a high-level SCI, dynamic CA is severely altered [22,23]. If CA fails, irreversible neuronal cell death can occur [24]. Therefore, the importance of CA for maintaining CBF during tilt has been recognized. A previous study has also assessed whether $\mathrm{CBF}$ increased when midodrine, a drug for treating $\mathrm{OH}$, was administered [25].

In the aforementioned studies of CBF in patients with an SCI, TCD was used to assess the flow velocities, but the cross-sectional areas of blood vessels could not be evaluated; therefore, there is a limit to measuring the exact CBFV with TCD. For this reason, we used CDU to measure blood flow, and we measured ICA BFV as an estimate of CBF. Previous studies have measured ICA BFV to estimate CBF [9-12], confirming that it shows a significant correlation with CBFV, compared to TCD [12]. To our knowledge, this study is the first to measure changes in CBF in patients with an SCI by performing CDU, and we found that presyncopal symptoms occurred when ICA blood flow was reduced by $\geq 21 \%$ with the patient in a tilted position. It was confirmed once again that $C A$, which modulates $\mathrm{CBF}$, is important in the presence or absence of $\mathrm{OH}$ symptoms. The incidence of $\mathrm{OH}$ after an $\mathrm{SCI}$ is related to the level of injury, degree of damage, time spent lying in bed, physical function, etc. The more severe the paralysis, the higher the incidence rate of $\mathrm{OH}$ [26]. Consistent with this, in our study, the physical and functional scores in the group in which $C A$ failed were significantly lower than those in the group in which CBF was maintained, and the proportion of AIS grades A, B, and C (i.e., the proportion of patients with a relatively severe SCI) was high in the former group. Regarding the correlation with presyncopal symptoms, we believe that $\mathrm{CBF}$ was preserved in $\mathrm{G} 2$, and the symptom-free rate was, therefore, high (only $5.56 \%$ had $\mathrm{OH}$ symptoms). In this group, even when $\mathrm{OH}$ was confirmed, there was no need to discontinue the rehabilitation treatment with tilt, and it was not necessary to administer drugs such as midodrine. Contrastingly, in G3, there was no decrease in BP during tilt, but CBF had decreased by more than $21 \%$ resulting in presyncopal symptoms in $40 \%$ of patients, which required the consideration for the administration of an anti-OH drug. Since CBF is often reduced in patients with $\mathrm{OH}$ symptoms due to $\mathrm{CA}$ failure, it is appropriate to measure $\mathrm{CBF}$ as well as $\mathrm{BP}$ in clinical practice, and to administer drugs if there is up to $21 \%$ decrease in CBF. Additionally, in clinical practice, patients with a high-level SCI often discontinue rehabilitation treatment, such as tilt table application due to reduction in BP. In these patients, if CA is ascertained by measuring $\mathrm{CBF}$ and there are no symptoms, treatment can be continued and progress can be observed even if there is a drop in BP. Overall, to confirm orthostatic tolerance, both $\mathrm{BP}$ and $\mathrm{CBF}$ should be assessed in order to properly implement the treatment for $\mathrm{OH}$.

\section{Limitations}

CDU was used to assess CBF in this study. Previous studies have measured regional CBF according to changes in BP using TCD, and some studies have shown that the ICA/MCA region is more sensitive to orthostatic challenges than the vertebral artery/posterior cerebral artery region $[25,27,28]$. Further studies are needed to determine the extent to which ICA blood flow can reflect total CBF. In addition, several factors, such as blood viscosity/hematocrit and intracranial pressure, may affect blood flow velocity, but these factors were not controlled in this study. Future studies will need to take these factors into consideration. When performing CDU, it is important to measure the cross-sectional area of the artery at a certain location and time. In this study, CDU was performed by more than one person; thus, there might have been inter-observer differences in the data obtained, depending on the proficiency and skills of the observers. Moreover, this study is a retrospective, non-blinded study; the examiner already knew about the patient's condition when performing the examination, and this may have introduced some bias. Furthermore, the differences in DOI were not considered. A future study that correlates changes in CBF with DOI in individual patients can help elucidate the cerebral hemodynamics over time. 
Patients with an SCI suffer from voiding problems, for which most of them take medications such as $\alpha$-blockers, which can cause OH. This study did not find out whether patients were taking such medications, and this should be considered in future studies. Another limitation is that only the presence or absence of presyncopal symptoms was noted; their severity, which would be more useful for determining the degree of orthostatic tolerance, was not considered. In order to obtain a more accurate conclusion, future studies should include more patients and have a longer follow-up period. There is no precise guideline on whether to administer an anti-OH drug if the SBP falls but the CBF is preserved. In a future study, the follow-up of changes in CBF and prognosis after treatment of $\mathrm{OH}$ will be helpful in establishing guidelines for further treatment.

\section{Conclusions}

This study used CDU to confirm changes in CBF during postural changes in patients with a CSCI. If CBFV decreased by more than $21 \%$, presyncopal symptoms occurred. However, even in the presence of $\mathrm{OH}$, if $\mathrm{CBFV}$ was preserved (i.e., if $\mathrm{CA}$ occurred), patients were less symptomatic. The higher the SCI severity and the lower the functional score, the higher the possibility of CA failure. It is, therefore, necessary to use CDU to assess CBF in patients with a high-level SCI, in order to ensure proper administration of drugs and smooth rehabilitation, and to ultimately improve the quality of life of patients.

Author Contributions: Study design: J.-H.K. and K.H.C.; data collection and analysis: J.-H.K., J.-H.H., C.-W.M. and K.H.C.; manuscript writing: J.-H.K. and K.H.C. All authors have read and agreed to the published version of the manuscript.

Funding: This research received no external funding.

Institutional Review Board Statement: The study was conducted according to the guidelines of the Declaration of Helsinki, and approved by the Institutional Review Board of the Chungnam National University Hospital (IRB No. 2020-05-091 and date of approval: 11 June 2020).

Informed Consent Statement: Patient consent was waived due to the retrospective nature of the study and because the analysis used anonymous clinical data.

Data Availability Statement: The data presented in this study are available from the corresponding author upon reasonable request.

Conflicts of Interest: The authors declare that the research was conducted in the absence of any commercial or financial relationships that could be construed as a potential conflict of interest.

\section{References}

1. Krassioukov, A.V.; Karlsson, A.K.; Wecht, J.M.; Wuermser, L.A.; Mathias, C.J.; Marino, R.J.; Joint Committee of American Spinal Injury Association; International Spinal Cord Society. Assessment of Autonomic Dysfunction Following Spinal Cord Injury: Rationale for Additions to International Standards for Neurological Assessment. J. Rehabil. Res. Dev. 2007, 44, 103-112. [CrossRef] [PubMed]

2. Carlozzi, N.E.; Fyffe, D.; Morin, K.G.; Byrne, R.; Tulsky, D.S.; Victorson, D.; Lai, J.S.; Wecht, J.M. Impact of Blood Pressure Dysregulation on Health-Related Quality of Life in Persons with Spinal Cord Injury: Development of a Conceptual Model. Arch. Phys. Med. Rehabil. 2013, 94, 1721-1730. [CrossRef]

3. Illman, A.; Stiller, K.; Williams, M. The prevalence of Orthostatic Hypotension during Physiotherapy Treatment in Patients with an Acute Spinal Cord Injury. Spinal Cord 2000, 38, 741-747. [CrossRef] [PubMed]

4. Claydon, V.E.; Steeves, J.D.; Krassioukov, A. Orthostatic Hypotension Following Spinal Cord Injury: Understanding Clinical Pathophysiology. Spinal Cord 2006, 44, 341-351. [CrossRef]

5. Phillips, A.A.; Krassioukov, A.V.; Ainslie, P.N.; Warburton, D.E. Baroreflex function after Spinal Cord Injury. J. Neurotrauma 2012, 29, 2431-2445. [CrossRef]

6. Gonzalez, F.; Chang, J.Y.; Banovac, K.; Messina, D.; Martinez-Arizala, A.; Kelley, R.E. Autoregulation of Cerebral Blood Flow in Patients with Orthostatic Hypotension after Spinal Cord Injury. Paraplegia 1991, 29, 1-7. [CrossRef]

7. Brass, L.; Prohovnik, I.; Pavlakis, S.; DeVivo, D.; Piomelli, S.; Mohr, J. Middle Cerebral Artery Blood Velocity and Cerebral Blood Flow in Sickle Cell Disease. Stroke 1991, 22, 27-30. [CrossRef]

8. Brooks, D.; Redmond, S.; Mathias, C.; Bannister, R.; Symon, L. The Effect of Orthostatic Hypotension on Cerebral Blood Flow and Middle Cerebral Artery Velocity in Autonomic Failure, with Observations on the Action of Ephedrine. J. Neurol. Neurosurg. Psychiatry 1989, 52, 962-966. [CrossRef] 
9. Rothoerl, R.; Schebesch, K.; Woertgen, C.; Brawanski, A. Internal Carotid Artery Volume Flow Correlates to Rcbf Measurements. Acta Neurochir. 2003, 145, 943-947. [CrossRef] [PubMed]

10. Soustiel, J.F.; Levy, E.; Zaaroor, M.; Bibi, R.; Lukaschuk, S.; Manor, D. A New Angle-Independent Doppler Ultrasonic Device for Assessment of Blood Flow Volume in the Extracranial Internal Carotid Artery. J. Ultrasound Med. 2002, 21, 1405-1412. [CrossRef] [PubMed]

11. Soustiel, J.; Glenn, T.; Vespa, P.; Rinsky, B.; Hanuscin, C.; Martin, N. Assessment of Cerebral Blood Flow by Means of Blood-FlowVolume Measurement in the Internal Carotid Artery: Comparative Study with a 133xenon Clearance Technique. Stroke 2003, 34, 1876-1880. [CrossRef] [PubMed]

12. Schebesch, K.-M.; Woertgen, C.; Schlaier, J.; Brawanski, A.; Rothoerl, R.D. Doppler Ultrasound Measurement of Blood Flow Volume in the Extracranial Internal Carotid Artery for Evaluation of Brain Perfusion after Aneurysmal Subarachnoid Hemorrhage. Neurol. Res. 2007, 29, 210-214. [CrossRef] [PubMed]

13. Claydon, V.E.; Krassioukov, A.V. Orthostatic Hypotension and Autonomic Pathways after Spinal Cord Injury. J. Neurotrauma 2006, 23, 1713-1725. [CrossRef]

14. Johnson, R.; Park, D.; Frankel, H. Orthostatic Hypotension and the Renin-Angiotensin System in Paraplegia. Spinal Cord 1971, 9, 146-152. [CrossRef] [PubMed]

15. Hetzel, A.; Reinhard, M.; Guschlbauer, B.; Braune, S. Challenging Cerebral Autoregulation in Patients with Preganglionic Autonomic Failure. Clin. Auton. Res. 2003, 13, 27-35. [CrossRef]

16. Krassioukov, A. Autonomic Function following Cervical Spinal Cord Injury. Resp. Physiol. Neurobiol. 2009, 169, 157-164. [CrossRef]

17. Osborn, J.W.; Taylor, R.F.; Schramm, L.P. Determinants of Arterial Pressure after Chronic Spinal Transection in Rats. Am. J. Physiol. Regul. Integr. Comp. Physiol. 1989, 256, R666-R673. [CrossRef] [PubMed]

18. Šerić, V.; Blažić-Čop, N.; Demarin, V. Haemodynamic Changes in Patients with Whiplash Injury Measured by Transcranial Doppler Sonography (TCD). Coll. Antropol. 2000, 24, 197-204.

19. Bleton, H.; Sejdić, E. A Cerebral Blood Flow Evaluation during Cognitive Tasks Following a Cervical Spinal Cord Injury: A Case Study Using Transcranial Doppler Recordings. Cogn. Neurodyn. 2015, 9, 615-626. [CrossRef]

20. Phillips, A.A.; Ainslie, P.N.; Krassioukov, A.V.; Warburton, D.E. Regulation of Cerebral Blood Flow after Spinal Cord Injury. J. Neurotrauma 2013, 30, 1551-1563. [CrossRef]

21. Willie, C.; Colino, F.; Bailey, D.; Tzeng, Y.; Binsted, G.; Jones, L.; Haykowsky, M.; Bellapart, J.; Ogoh, S.; Smith, K. Utility of Transcranial Doppler Ultrasound for the Integrative Assessment of Cerebrovascular Function. J. Neurosci. Methods 2011, 196, 221-237. [CrossRef] [PubMed]

22. Sahota, I.S.; Ravensbergen, H.J.; McGrath, M.S.; Claydon, V.E. Cerebrovascular Responses to Orthostatic Stress after Spinal Cord Injury. J. Neurotrauma 2012, 29, 2446-2456. [CrossRef] [PubMed]

23. Wilson, L.C.; Cotter, J.D.; Fan, J.-L.; Lucas, R.A.; Thomas, K.N.; Ainslie, P.N. Cerebrovascular Reactivity and Dynamic Autoregulation in Tetraplegia. Am. J. Physiol. Regul. Integr. Comp. Physiol. 2010, 298, R1035-R1042. [CrossRef] [PubMed]

24. Aries, M.J.; Elting, J.W.; De Keyser, J.; Kremer, B.P.; Vroomen, P.C. Cerebral Autoregulation in Stroke: A Review of Transcranial Doppler Studies. Stroke 2010, 41, 2697-2704. [CrossRef] [PubMed]

25. Phillips, A.A.; Krassioukov, A.V.; Ainslie, P.N.; Warburton, D.E. Perturbed and Spontaneous Regional Cerebral Blood Flow Responses to Changes in Blood Pressure after High-level Spinal Cord Injury: The effect of midodrine. J. Appl. Physiol. 2014, 116, 645-653. [CrossRef] [PubMed]

26. Sidorov, E.; Townson, A.; Dvorak, M.; Kwon, B.; Steeves, J.; Krassioukov, A. Orthostatic Hypotension in the First Month following Acute Spinal Cord Injury. Spinal Cord 2008, 46, 65-69. [CrossRef]

27. Azevedo, E.; Castro, P.; Santos, R.; Freitas, J.; Coelho, T.; Rosengarten, B.; Panerai, R. Autonomic Dysfunction Affects Cerebral Neurovascular Coupling. Clin. Auton. Res. 2011, 21, 395-403. [CrossRef]

28. Sato, K.; Fisher, J.P.; Seifert, T.; Overgaard, M.; Secher, N.H.; Ogoh, S. Blood Flow in Internal Carotid and Vertebral Arteries during Orthostatic Stress. Exp. Physiol. 2012, 97, 1272-1280. [CrossRef] 